\title{
Antagonistic regulation of Drosophila mitochondrial uncoupling protein UCP4b by cold and BMP signaling
}

Nirmalya Chatterjee ${ }^{1, \star}$, Wei Song ${ }^{1,5}$, Phillip A. Dumesic ${ }^{2,3}$, Bruce Spiegelman ${ }^{2,3}$, and Norbert Perrimon ${ }^{1,4, *}$

${ }^{1}$ Department of Genetics, Blavatnik Institute, Harvard Medical School, Boston, MA 02115, USA

${ }^{2}$ Dana-Farber Cancer Institute, Boston, MA 02115, USA

${ }^{3}$ Department of Cell Biology, Blavatnik Institute, Harvard Medical School, Boston, MA 02115, USA

${ }^{4}$ Howard Hughes Medical Institute, Boston, MA 02115, USA

${ }^{5}$ Frontier Science Center for Immunology and Metabolism, Medical Research Institute, Wuhan University, Wuhan, Hubei 430071, China

${ }^{*}$ Corresponding authors:

Nirmalya Chatterjee: Nirmalya_Chatterjee@hms.harvard.edu

Norbert Perrimon: perrimon@genetics.med.harvard.edu

\begin{abstract}
Regulation of energy metabolism and response to cold are intimately linked in mammals. Central to these two processes are the mitochondrial uncoupling proteins (UCPs), which by promoting proton leakage across the inner mitochondrial membrane lead to the generation of heat instead of ATP synthesis. In addition to heat generation, UCPs also influence energy storage and can protect against obesity and diabetes. Cold-blooded animals like flies also contain UCPs that protect from cold, however their regulations are poorly understood. We find that Drosophila UCP4b is induced by cold in a cell-intrinsic manner and protects against cold and obesity in fly models. Mechanistically, cold regulates UCP4b expression through calcium signaling and Spargel (Srl), the Drosophila ortholog of mammalian PGC1 $\alpha$. To the opposite, $M A D$, acting downstream of the BMP branch of the TGF $\beta$ signaling pathway, represses UCP $4 b$ expression independently of cold. Interestingly, the two mechanisms of $U C P 4 b$ regulation are integrated as MAD binding to the $U C P 4 b$ promoter is displaced by cold in a Srl-dependent manner. We discuss the similarities between the regulation of mammalian and Drosophila UCPs.
\end{abstract}

\section{Significance}

Mitochondrial uncoupling proteins (UCPs) that uncouple the mitochondrial respiration from ATP synthesis regulate energy metabolism, non-shivering thermogenesis, and redox balance in vertebrates and invertebrates. However, their regulation in Drosophila is poorly understood. We 
found that Drosophila uncoupling protein UCP4b is induced by cold in a cell-autonomous fashion. Conversely, MAD, acting downstream of BMP signaling, inhibits UCP $4 b$ expression. MAD is displaced from the upstream regions of the UCP $4 b$ gene by cold. UCP $4 b$ protects Drosophila against cold and diet-induced obesity. The regulation of UCP4b by cold and BMP signaling is reminiscent of the regulation of mammalian uncoupling protein UCP1. Altogether, we discovered an important regulator of Drosophila energy metabolism which is controlled by regulatory processes that are similar between Drosophila and mammals.

\section{Introduction}

Mitochondrial uncoupling proteins (UCPs), a family of transmembrane proton carriers, can cause proton leakage across the inner mitochondrial membrane and dissipate the proton gradient generated by the electron transport chain in the form of heat. This dissociates mitochondrial respiration from the function of ATP synthase that uses the proton gradient to generate ATP (1). UCPs play important roles in non-shivering thermogenesis (NST), a method of conversion of metabolic energy into heat without skeletal muscle contraction. In particular, mammalian mitochondrial uncoupling protein UCP1 (thermogenin), which is induced in brown adipose tissue (BAT) in response to cold, plays a pivotal role in NST (2). In addition to thermoregulation, mammalian UCPs reduce mitochondrial reactive oxygen species (ROS) generation by reducing the proton gradient across the inner mitochondrial membrane. For example, UCP2 expression is reduced in macrophages with a concomitant increase in ROS that is required to prevent infection (3), whereas UCP3 protects the heart against ischemiareperfusion (IR) injury, which causes oxidative stress (4). Similarly, reduction in the expression of UCP4 and UCP45 in the dopaminergic neurons is implicated in oxidative stress in the DJ-1 (PARK7) mouse model of Parkinson's disease (5).

The regulation of the expression of mammalian Ucp1 by cold is fairly well characterized. Norepinephrine released in response to cold exposure triggers $\beta$-adrenergic signaling in brown adipose tissue (BAT), leading to the induction of Ucp1 expression in BAT (2). In addition, the transcriptional co-activator Ppargc1a is induced in the mammalian BAT by cold-triggered $\beta$ adrenergic signaling and is involved in cold-mediated Ucp1 induction in the BAT (6). The absence of PGC1 $\alpha$ in brown adipocytes impairs the activation of the thermogenic gene expression program in response to cold (7). Interestingly, cold exposure can also increase thermogenic gene expression including Ucp1 and Ppargc1a in white and beige adipocytes in a cell-autonomous fashion (8). This yet uncharacterized mechanism is different from the coldmediated Ucp1 induction in BAT (8). On the other hand, TGF $\beta$ /BMP signaling suppresses mammalian Ucp1 expression. Smad3, acting downstream of TGF $\beta$ signaling, represses Ucp1 expression in mammalian white adipose tissue (WAT) (9). Moreover, the induction of Ucp1 under Smad3 loss-of-function condition is also accompanied by an induction of Ppargc1a. Interestingly, Smad3 deficiency prevents diet-induced obesity in mice (9). Similarly, BMP4, which promotes a brown to white-like adipocyte shift, inhibits Ucp1 expression (10). Interestingly, BMP4 also suppresses Ppargc1a expression (10).

Insects, being cold-blooded animals employ processes such as cold avoidance, accumulation of cryoprotectants in the hemolymph, and changes in the membrane composition in response to cold (11-13). However, recent studies have indicated that mechanisms similar to mammalian non-shivering thermogenesis are also involved in cold tolerance in insects. There are four mitochondrial UCPs in Drosophila, namely UCP4a, UCP4b, UCP4c, and UCP5 (14). UCP4c 
promotes mitochondrial uncoupling and is required for the development of larvae when raised at cold temperatures (15). In addition, the expression of UCP4b and UCP4c is suppressed by the circadian rhythm master-regulator gene period (per). per mutants show a high metabolic rate due to mitochondrial uncoupling and are more tolerant to cold. Interestingly, disrupted expression of UCP $4 \mathrm{~b}$ and UCP4c reverts the increase in mitochondrial respiration and enhanced cold tolerance in per mutant flies (16). Consistent with redox-regulatory effects of mammalian UCPs, Drosophila UCPs are also implicated in redox regulation. The periodic expression of UCP4b and UCP4c orchestrated by per reduces the ROS load in Drosophila intestinal stem cells (ISCs) and maintains their proliferative homeostasis (16). Moreover, UCP4a overexpression in the pink1/parkin Drosophila models of Parkinson's disease reduce ROS levels and protect against oxidative stress (17). Thus, like mammalian UCPs, Drosophila UCPs are also involved in both cold tolerance and redox regulation indicating a conservation of physiological functions of UCPs between Drosophila and mammals. In addition to Drosophila UCPs, Spargel (Srl), similar to its mammalian ortholog PGC1 $\alpha$, has also been implicated in cold tolerance as a mutation in Srl renders Drosophila susceptible to cold (18).

While UCP-mediated non-shivering thermogenesis mechanism is functional in Drosophila, it is not known how Drosophila UCPs are regulated. Here, we report that Drosophila UCP $4 b$ is induced in a cell-autonomous fashion by cold and is suppressed by BMP signaling. Moreover, we found that Srl plays a central role in the regulation of UCP $4 b$ by both cold and BMP signaling. Our study demonstrates that the role of some proteins and signaling pathways involved in the regulation of mitochondrial bioenergetics is generally evolutionarily conserved between Drosophila and mammals.

\section{Results}

UCP4b is induced by cold in a cell-autonomous fashion: To determine if Drosophila UCPs, like mammalian UCP1, can be induced by cold, we tested the expression of UCPs after cold exposure of different durations. Strikingly, only $U C P 4 b$ is induced in response to cold exposure in adult flies (Fig 1A), and overexpression of UCP4b protects against cold (Fig 1B), whereas UCP4b knockdown makes them more susceptible to cold (Fig 1C). Similarly, UCP4b is significantly induced in Drosophila larvae in response to cold exposure (Fig 1D), and only $U C P 4 b$ is induced by cold in multiple larval tissues such as the adipose tissue (the fat body), the skeletal muscle (the body wall), the gut, and the brain (Fig 1E, Fig S1, S2). Interestingly, UCP4b is also induced by cold in S2R+ and Kc Drosophila cells (Fig 1F, Fig S3), indicating that these cells can directly sense cold and can induce UCP4b independently of any neuronal input. Thus, cold induces UCP $4 b$ transcription in adult flies, multiple larval tissues, and in cultured Drosophila cells. Both mammalian and Drosophila UCPs have been shown to cause mitochondrial depolarization $(2,15,16)$. To test the effect of UCP4b on mitochondrial potential, we generated a stable S2R + cell line expressing UCP4b under a metal-inducible promoter. These cells were stained with TMRM which accumulates in mitochondria with higher membrane potential. Copper sulfate induced UCP4b overexpression reduced the TMRM signal indicating mitochondrial depolarization (Fig 1G). Interestingly, genetic and pharmacological upregulation of UCP1 reduces obesity in mammals (19). Similarly, ubiquitous expression of UCP4b in adults protects against high sugar-diet (HSD) induced increase in TAG levels (Fig. 1H).

Calcium signaling and Spargel (Srl) are involved in the cold-mediated UCP4b induction: Calcium signaling has been implicated in brain-independent ex vivo cold-sensing and rapid cold hardening $(\mathrm{RCH})$ in insect tissues (20). Thus, we tested the role of calcium signaling in the coldmediated induction UCP $4 b$ by monitoring the activity of the genetically encoded calcium 
indicator GCaMP in dissected larval fat body. ex vivo application of cold to larval adipose tissue activates calcium signaling (Fig 2A; Supplementary movie S1) and induces UCP4b (Fig S4). Moreover, pre-treatment with the chelator BAPTA-AM attenuates cold-mediated UCP4b induction in S2R+ cells indicating that calcium signaling plays a role in cold-mediated induction of UCP $4 b$ (Fig 2B). Cold-mediated UCP4b induction is not affected by pre-treatment with cycloheximide indicating that no intermediate protein synthesis is required for the process (Fig 2C). Moreover, UCP4b induction is completely attenuated by pre-treatment with actinomycin D (Fig 2D), indicating that cold-mediated increase in UCP4b transcript levels is principally caused by enhanced transcription of the UCP4b gene. Interestingly, knockdown of Srl, the ortholog of mammalian transcriptional co-activator Ppargc1a, strongly suppressed cold-mediated UCP4b induction in S2R+ cells (Fig 2E). However, Srl is not induced in response to cold either in cultured S2R+ cells (Fig 2F) nor in adult flies (Fig S5). Interestingly, ubiquitous Srl overexpression in larvae is sufficient to induce UCP4b in whole larvae (Fig 2G). Moreover, consistent with a previous study (18), Srl knockdown renders flies more susceptible to cold (Fig $2 \mathrm{H})$. Thus, both calcium signaling and Srl are required for cold-mediated UCP $4 b$ induction.

UCP4b is suppressed by BMP signaling: MAD and its interacting partner MED, which is the ortholog of mammalian SMAD4, have been shown to bind to the upstream regions of UCP4b (21). These prompted us to explore the role of BMP signaling in the regulation of UCP4b. Interestingly, knockdown of MAD in S2R+ cells as well as knockdown of other components of BMP signaling such as MED, Type I receptors Tkv and Sax, Type II receptor Put, and Gbb, the only BMP ligand expressed in S2R+ cells, induced UCP4b expression under regular culture conditions at $25^{\circ} \mathrm{C}$ (Fig 3A). This indicates that BMP signaling suppresses UCP4b under basal conditions in both Drosophila larval fat body and in cultured cells. Moreover, the application of LDN212854, which has been reported to inhibit mammalian BMP signaling (22), leads to rapid induction of UCP4b expression in S2R+ cells (Fig 3B). This LDN212854-mediated UCP4b induction is not affected by a pre-treatment with cycloheximide $(\mathrm{CHX})$, indicating that no intermediate protein synthesis is required for the process (Fig $3 \mathrm{C}$ ). In addition, UCP4b was induced in adult flies fed a diet containing LDN212854 (Fig. 3D). On the other hand, ubiquitous overexpression of $M A D$ in larvae suppressed cold-induced $d U C P 4 b$ expression (Fig $3 \mathrm{E}$ ). Similarly, MAD overexpression in the larval fat body suppressed UCP4b expression in the fat body (Fig 3F). Similar to the cold-mediated UCP4b induction, the induction of UCP4b in response to BMP signaling inhibition is dependent on Srl but $\mathrm{Srl}$ is not induced under these conditions (Fig $3 \mathrm{G}, \mathrm{H}$ ), indicating that Srl plays a central role in the regulation of UCP4b expression by both cold and BMP signaling.

Crosstalk between BMP signaling and cold in the regulation of UCP4b: To understand the crosstalk between BMP signaling and cold in the regulation of UCP $4 b$, we explored the molecular changes in response to cold exposure as well as to the pharmacological inhibition of BMP signaling. Consistent with its role in mammalian cells, LDN212854 reduces MAD phosphorylation triggered by Tkv and Tkv act overexpression in S2R+cells, thus establishing that LDN212854 can inhibit BMP signaling in Drosophila (Fig 4A). Interestingly, unlike LDN212854, cold exposure did not change Tkv and $\mathrm{Tkv}^{\text {act }}$ overexpression-driven MAD phosphorylation indicating that BMP signaling upstream of MAD phosphorylation is not affected by cold (Fig 5A), suggesting that UCP4b is induced through different mechanisms by cold and LDN212854. This model is supported by the observation that simultaneous application of cold and LDN212854 synergistically induces $U C P 4 b$ (Fig 4B). Furthermore, MAD knockdown also results in synergistic induction of UCP4b expression when combined with cold exposure (Fig 4C). Interestingly, both cold exposure and LDN212854 reduce MAD binding to the upstream regions of the UCP4b gene suggesting that cold exposure removes the inhibitory effect of BMP signaling on UCP4b by displacing MAD from the upstream regions of UCP4b (Fig 4D). 
Moreover, this displacement of MAD by cold is abrogated by Srl knockdown, suggesting that Srl is required for cold-mediated displacement of MAD from the upstream regions of UCP $4 b$ (Fig $4 \mathrm{E})$. Consistent with the synergistic induction of UCP4b by LDN212854 and cold, flies fed a food containing LDN212854 are better protected against cold compared to the flies on control food (Fig 4F). Moreover, this protection was attenuated by Srl knockdown (Fig 4F). Thus, the transcriptional repression of UCP4b by BMP signaling under basal conditions is removed by cold acting through Srl, resulting in UCP4b induction.

\section{Discussion}

We report that, like mammalian Ucp1, Drosophila UCP4b is induced by cold, is suppressed by BMP signaling, and plays a role in cold tolerance and energy storage. Our studies indicate that at normal temperature, MAD, acting downstream of BMP signaling, binds to the upstream regions of the $U C P 4 b$ gene and suppresses its transcription. Cold exposure acts through Srl to displace MAD and promote UCP4b transcription (Fig. 4G). MAD overexpression stoichiometrically counters this displacement and thereby partially blunts the cold-mediated UCP4b induction (as seen in Fig. 3E). On the other hand, MAD knockdown or LDN-mediated BMP signaling inhibition reduces this inhibitory component and thereby facilitates UCP $4 b$ induction (as seen in Fig. 3A and 3B). The inducing effect of cold on UCP4b expression synergizes with the effect of either MAD knockdown or LDN-mediated BMP inhibition to cause a stronger UCP4b induction (as seen in Fig. 4B and 4C). In addition, calcium signaling is triggered in response to cold and is important for cold-mediated UCP4b induction.

Recent studies have shown that non-shivering thermogenesis plays a role in cold tolerance in Drosophila. Mammalian Ucp1, which is induced in response to cold, plays a crucial role in nonshivering thermogenesis (2). Studies in Drosophila have shown that UCPs play a similar role in flies. UCP4C knockdown renders larvae sensitive to cold (15) whereas disrupted expression of $U C P 4 b$ and UCP4C due to transposon insertion reduces cold tolerance in adult flies with per deficiency (16). Moreover, UCP-independent non-shivering thermogenesis is also shared between mammals and Drosophila. Sarcolipin uncouples ATP hydrolysis from $\mathrm{Ca}^{2+}$ transport into the endoplasmic reticulum (ER) lumen by interacting with Sarco/ER $\mathrm{Ca}^{2+}$ adenosine triphosphatase (SERCA) in mammalian muscles (23). Similarly, thyroid adenoma associated (THADA) uncouples ATP hydrolysis from the $\mathrm{Ca}^{2+}$ transport conducted by SERCA in Drosophila. This leads to reduced $\mathrm{Ca}^{2+}$ transport into the $\mathrm{ER}$, and as a consequence, the energy from ATP hydrolysis is dissipated in the form of heat. Consistent with this observation, THADAdeficient flies have reduced energy production, are hyperphagic and obese, and are sensitive to cold (24). Thus, multiple mechanisms of non-shivering thermogenesis are shared between mammals and flies. Adding to these previous studies, we have shown that in addition to the evolutionarily conserved role in cold tolerance, Drosophila UCP4b, like mammalian Ucp1 is also induced by cold.

The regulation of mammalian Ucp1 by TGF $\beta$ /BMP signaling is complex. On one hand, Smad3, acting downstream of TGF $\beta$ signaling suppresses Ucp1 expression in the white adipose tissue (9). Moreover, Smad3 binds to the promoter region of Ppargc1a, a transcriptional inducer of Ucp1, and represses Ppargc1a transcription in white adipocytes (9). Similarly, BMP4 signaling suppresses Ucp1 expression in brown pre-adipocytes (10). Interestingly, BMP4 also represses the transcription of Ppargc1a in brown pre-adipocytes (10). On the other hand, BMP7 promotes the expression of $U_{c p} 1$ and regulators of brown fat fate including Ppargc1a in brown preadipocytes (25). Similarly, TGF- $\beta 2$, an exercise-induced adipokine, promotes the expression of 
both in Ucp1 and Ppargc1a BAT (26). Moreover, hepatic overexpression of activin E promotes Ucp1 expression in inguinal WAT, mesenteric WAT and interscapular BAT (27). In contrast, hepatic overexpression of activin E promotes Ppargc1a expression only in interscapular BAT but not in inguinal WAT and mesenteric WAT (27). Thus, various TGF $\beta$ and BMP ligands have different effects on Ucp1 expression in different adipose tissues. Moreover, the induction of Ucp 1 is mirrored by an induction of Ppargc1a in many cases but not all the time. Here, we report that, as in mammals BMP signaling regulates the expression of a Drosophila UCP (UCP4b), highlighting the evolutionary conservation of this mechanism.

We have demonstrated that there are similarities between the regulation of mammalian and Drosophila UCPs. Similar to the mammalian Ucp1, Drosophila UCP4b is induced by cold. Interestingly, we found that $U C P 4 b$ is induced by cold in a cell-intrinsic fashion, which is reminiscent of the cell-autonomous induction of Ucp1 in mammalian white adipocytes (8). Ppargc1a is important for transcriptional activation of Ucp1 in BAT in response to cold (6). Similarly, we have found that PGC1 $\alpha$ is required for cold-mediated induction of UCP4b. In contrast, TGF $\beta / B M P$ signaling represses mammalian Ucp1 expression $(9,10)$. Consistent with that observation, we found that BMP signaling also represses UCP4b expression in Drosophila. Thus, the antagonistic pattern of regulatory effects of cold and BMP signaling on UCPs is shared between Drosophila and mammals. However, we found differences between the regulation of mammalian Ucp1 and Drosophila UCP4b as well. Ppargc1a is induced by coldinduced $\beta$-adrenergic stimulation in BAT and promotes Ucp1 induction (6). Similarly, Ppargc1a is also induced by cold cell-autonomously in white adipocytes (8). However, Drosophila Srl was not induced by cold. Interestingly, Ppargc1a is suppressed by both Smad3, the effector of TGF $\beta$ signaling and BMP4 in WAT and brown pre-adipocytes, respectively $(9,10)$. However, we found that, although $\mathrm{Srl}$ is required for UCP4b induction under BMP signaling loss-of-function conditions, it is not induced under those conditions. These observations highlight that, while the regulatory networks controlling UCPs share similarities between flies and mammals, the detailed molecular mechanism has partially diverged through evolution.

It will be important to elucidate how Drosophila cells sense cold and trigger calcium signaling. The observation that Srl is important for cold-mediated UCP4b induction, but is not induced by cold, combined with the finding that cold-mediated UCP $4 b$ induction is not affected by cycloheximide-mediated inhibition of protein-synthesis, indicates that post-translational modification of Srl might be involved in cold-mediated UCP4b induction. Alternatively, posttranslational modification in interacting partners of Srl could also play role in this context. Posttranslational modifications of PGC1 $\alpha$, in addition to its cold-mediated induction, play a role in Ucp1 induction in mammals (28). Future studies in Drosophila will illuminate whether similar processes are involved in cold-mediated induction of UCP4b. Finally, as we found that LDN212854, a BMP signaling inhibitor synergistically induces UCP4b when combined with cold exposure, it will be of interest to determine how these signals interact and influence Ucp1 expression in mammalian WAT and mammalian energy homeostasis.

\section{Materials and method}

Fly strains, food, and cold treatment: Drosophila were raised in a humidity-controlled incubator at $25^{\circ} \mathrm{C}$ with $12 / 12 \mathrm{hr}$ dark/light cycles on standard lab food containing $15 \mathrm{~g}$ yeast, 8.6 $\mathrm{g}$ soy flour, $63 \mathrm{~g}$ cornmeal, $5 \mathrm{~g}$ agar, $5 \mathrm{~g}$ malt, $74 \mathrm{~mL}$ corn syrup per liter. A high-sugar diet (15 $\mathrm{w} / \mathrm{v}$ HSD) was prepared by adding $10 \mathrm{~g}$ of Sucrose to $50 \mathrm{~g}$ of standard lab food. LDN-food was prepared by mixing LDN-212854 with the standard lab food to a final concentration of $200 \mathrm{uM}$. 
Young adult flies were fed on this food for 6 days before RNA extraction and qPCR analysis. The cold sensitivity assay was carried out by exposing young adult flies in food vials ( 3 replicates, 20 each, sexes separated after mating) to $4^{\circ} \mathrm{C}$ for 6 days and then were brought back to $25^{\circ} \mathrm{C}$ and immediately transferred to new vials. Survivors were scored after 4 hours. The following Drosophila strains were obtained from the Bloomington Drosophila Stock Center (BDSC): UCP4b-RNAi (66999), Srl-RNAi (33914, 33915). The Srl overexpression line is described by Rera et al (29). We generated the UCP4b overexpression fly line by integrating a plasmid expressing UCP4b-RA isoform with C-terminus HA tag under UAS promoter into the attP2 site.

RT-qPCR: mRNA was prepared from whole flies, larvae, larval tissue, and from S2R+ cells using Trizol (Invitrogen) followed by DNase (TurboDNA free kit, Invitrogen) treatment and RNA purification with "RNeasy kit" (Qiagen). cDNA was synthesized using iScript cDNA Synthesis Kit (Bio-Rad). Real-time quantitative PCR (RT-qPCR) experiments were carried out using the Biorad CFX 96/384 machine. The iQ SYBR green supermix was used following the manufacturer's protocol. 'Delta-delta Ct' method was used to calculate fold change in experimental conditions. Three biological replicates were used and the transcript levels were normalized to Drosophila $\alpha$-tubulin and RpL32. The sequences of primers used in qPCR are provided in Supplementary Dataset S1.

Cell culture, transfection, dsRNA treatment, and drug treatment: Drosophila S2R+ cells were cultured in Schneider's medium with $10 \%$ fetal bovine serum (FBS) at $25^{\circ} \mathrm{C}$. S2R+ cells were transfected with plasmid DNA with Effectene transfection reagent (Qiagen) following the manufacturer's protocol. The plasmids expressing FLAG-tagged MAD, Tkv, and activated Tkv were a generous gift from Dr. Edward Eivers (30). A UCP4b overexpression stable cell line was generated by integrating a plasmid expressing UCP4b-RA isoform with a C-terminus HA tag under the metallothionine promoter into S2R+ cells. Knockdown of specified genes in S2R+ cells was achieved through treatment with dsRNAs that were synthesized using the MEGAscript T7 Transcription Kit (Invitrogen) and purified with "RNeasy kit" (Qiagen). S2R+ cells were treated with dsRNAs following the 'bathing' method (https://fgr.hms.harvard.edu/drsc-cell-rnai). Cells were treated with LDN-212854 (Sigma), cycloheximide (Sigma), actinomycin D (Calbiochem), and BAPTA-AM (Sigma) at specified concentrations and for the indicated time points. Primer sequences used for dsRNA-mediated RNA Interference are provided in Supplementary Dataset S2.

TAG assay: The specified number of animals or dissected tissue were homogenized in 250uL cold PBS/0.05\% Triton-X (PBS-TX) with $1 \mathrm{~mm}$ Zirconium beads (Next Advance, ZROB10) using Bullet Blender (Next Advance). Cell debris were removed using centrifugation and TAG level was measured with Infinity Triglyceride Reagent (Thermo Fisher Scientific). 5uL of homogenate or glycerol standard (Sigma) was added to $150 \mathrm{uL}$ of Triglyceride Reagent for each replicate in a 96-well plate. After 10 min incubation at $37^{\circ} \mathrm{C}$, the absorbance at $520 \mathrm{~nm}$ was recorded using a plate reader. The same homogenate was used for protein estimation using the Pierce BCA assay kit (Thermo Fisher Scientific). 2uL of homogenate or albumin standard (Thermo Fisher Scientific) was added to $200 \mathrm{uL}$ Pierce BCA solution for each replicate in a 96-well plate. After 30 min incubation at $37^{\circ} \mathrm{C}$, the absorbance at $562 \mathrm{~nm}$ was recorded using a plate reader.

TMRM assay: Transfected and copper sulfate (100uM)- or mock-treated cells were washed with PBS and plated in 96-well plates. Then cells were treated with 2 uM oligomycin A (Sigma) and stained with 10nM Tetramethylrhodamine, Methyl Ester, Perchlorate (TMRM) (Thermo Fisher Scientific) in complete Schneider medium for 30 minutes at $25^{\circ} \mathrm{C}$. Next, cells were 
washed with PBS and imaged using GE IN Cell Analyzer 6000 Cell Imaging System. 3 random fields were chosen for each of the 3 biological replicates for each condition.

Western blot: Transfected and subsequently cold- or LDN-212854-treated S2R+ cells were directly boiled in SDS sample buffer, run on a 4\%-20\% polyacrylamide gel (Bio-Rad), and then transferred to an Immobilon-P polyvinylidene fluoride (PVDF) membrane (Millipore). The membrane was blocked with $5 \%$ BSA in TBST (TBS with $0.1 \%$ Tween-20) at room temperature for 1 hour and then probed with the primary antibody in 1X TBST with 5\% BSA overnight. This was followed by incubation with an HRP-conjugated secondary antibody. The signal was detected by chemiluminescence (Thermo Fisher Scientific). Drosophila phospho-MAD was detected using an anti-Smad3 antibody (Abcam, ab52903), MAD-FLAG was detected using anti-FLAG-MAb (Sigma, F3165) whereas a-tubulin was detected using anti-alpha-tubulin MAb (Sigma, T5168).

ChIP: Chromatin immunoprecipitation (ChIP) was performed using SimpleChIP® Plus Enzymatic Chromatin IP Kit (Cell Signaling Technology, 9005S, Magnetic Beads) following the manufacturer's protocol. The chromatin from S2R+ cells that were transfected with Act>Gal4 and UAS-MAD-FLAG plasmids and treated in specified combinations of dsRNAs, drugs, and cold was sheared using a Bioruptor (Diagenode) at high frequency for 30 cycles of $30 \mathrm{~s}$ ON, 30 s OFF. The chromatin was precipitated using anti-FLAG-MAb (Sigma, F3165). The relative enrichment of the purified DNA was analyzed using qPCR with primers targeting the upstream region of the UCP4b gene. Primers (NC) targeting a gene desert on chromosome $2 \mathrm{R}$ of Drosophila melanogaster were used as a negative control (31). The sequences of primers used in qPCR are provided in Supplementary Dataset S1.

Acknowledgments: We are grateful to Dr. Edward Eivers for reagents. We thank Muhammad Ahmad and Sudhir Tattikota for help with microscopy, and Christians Villalta for fly embryo injections. This work was supported by NIH grants 5P01CA120964 and 5R01DK121409 to NP, NIH grants R01DK123228 and R01 DK119117 to BMS and DRCRF Fellowship DRG- 120-17 to PAD. NP is an investigator of HHMI.

\section{References}

1. Lowell BB \& Spiegelman BM (2000) Towards a molecular understanding of adaptive thermogenesis. Nature 404(6778):652-660.

2. Chouchani ET, Kazak L, \& Spiegelman BM (2019) New Advances in Adaptive Thermogenesis: UCP1 and Beyond. Cell Metab 29(1):27-37.

3. Ledesma A, de Lacoba MG, \& Rial E (2002) The mitochondrial uncoupling proteins. Genome Biol 3(12):REVIEWS3015.

4. Cadenas S (2018) Mitochondrial uncoupling, ROS generation and cardioprotection. Biochim Biophys Acta Bioenerg 1859(9):940-950.

5. Dolgacheva LP, Berezhnov AV, Fedotova EI, Zinchenko VP, \& Abramov AY (2019) Role of DJ-1 in the mechanism of pathogenesis of Parkinson's disease. J Bioenerg Biomembr 51(3):175-188. 
6. Collins S, Cao W, \& Robidoux J (2004) Learning new tricks from old dogs: betaadrenergic receptors teach new lessons on firing up adipose tissue metabolism. $\mathrm{Mol}$ Endocrinol 18(9):2123-2131.

7. Gill JA \& La Merrill MA (2017) An emerging role for epigenetic regulation of Pgc-1alpha expression in environmentally stimulated brown adipose thermogenesis. Environ Epigenet 3(2):dvx009.

8. Ye L, et al. (2013) Fat cells directly sense temperature to activate thermogenesis. Proc Natl Acad Sci U S A 110(30):12480-12485.

9. Yadav H, et al. (2011) Protection from obesity and diabetes by blockade of TGFbeta/Smad3 signaling. Cell Metab 14(1):67-79.

10. Modica S, et al. (2016) Bmp4 Promotes a Brown to White-like Adipocyte Shift. Cell Rep 16(8):2243-2258.

11. Overgaard J \& MacMillan HA (2017) The Integrative Physiology of Insect Chill Tolerance. Annu Rev Physiol 79:187-208.

12. Olsson T, et al. (2016) Hemolymph metabolites and osmolality are tightly linked to cold tolerance of Drosophila species: a comparative study. J Exp Biol 219(Pt 16):2504-2513.

13. Kostal V, et al. (2011) Long-term cold acclimation extends survival time at 0 degrees $C$ and modifies the metabolomic profiles of the larvae of the fruit fly Drosophila melanogaster. PLoS One 6(9):e25025.

14. Hanak P \& Jezek P (2001) Mitochondrial uncoupling proteins and phylogenesis--UCP4 as the ancestral uncoupling protein. FEBS Lett 495(3):137-141.

15. Da-Re C, et al. (2014) UCP4C mediates uncoupled respiration in larvae of Drosophila melanogaster. EMBO Rep 15(5):586-591.

16. Ulgherait $M$, et al. (2020) Circadian regulation of mitochondrial uncoupling and lifespan. Nat Commun 11(1):1927.

17. Wu K, Liu J, Zhuang N, \& Wang T (2014) UCP4A protects against mitochondrial dysfunction and degeneration in pink1/parkin models of Parkinson's disease. FASEB J 28(12):5111-5121.

18. Cobb T, Damschroder D, \& Wessells R (2021) Sestrin regulates acute chill coma recovery in Drosophila melanogaster. Insect Biochem Mol Biol 133:103548.

19. Kozak LP \& Anunciado-Koza R (2008) UCP1: its involvement and utility in obesity. Int J Obes (Lond) 32 Suppl 7:S32-38.

20. Teets NM, Yi SX, Lee RE, Jr., \& Denlinger DL (2013) Calcium signaling mediates cold sensing in insect tissues. Proc Natl Acad Sci U S A 110(22):9154-9159.

21. MacArthur S, et al. (2009) Developmental roles of 21 Drosophila transcription factors are determined by quantitative differences in binding to an overlapping set of thousands of genomic regions. Genome Biol 10(7):R80.

22. Dinter T, Bocobo GA, \& Yu PB (2019) Pharmacologic Strategies for Assaying BMP Signaling Function. Methods Mol Biol 1891:221-233.

23. Bal NC, et al. (2012) Sarcolipin is a newly identified regulator of muscle-based thermogenesis in mammals. Nat Med 18(10):1575-1579.

24. Moraru A, et al. (2017) THADA Regulates the Organismal Balance between Energy Storage and Heat Production. Dev Cell 41(1):72-81 e76. 
25. Tseng $\mathrm{YH}$, et al. (2008) New role of bone morphogenetic protein 7 in brown adipogenesis and energy expenditure. Nature 454(7207):1000-1004.

26. Takahashi $\mathrm{H}$, et al. (2019) TGF-beta2 is an exercise-induced adipokine that regulates glucose and fatty acid metabolism. Nat Metab 1(2):291-303.

27. Hashimoto O, et al. (2018) Activin E Controls Energy Homeostasis in Both Brown and White Adipose Tissues as a Hepatokine. Cell Rep 25(5):1193-1203.

28. Cao W, et al. (2004) p38 mitogen-activated protein kinase is the central regulator of cyclic AMP-dependent transcription of the brown fat uncoupling protein 1 gene. Mol Cell Biol 24(7):3057-3067.

29. Rera $M$, et al. (2011) Modulation of longevity and tissue homeostasis by the Drosophila PGC-1 homolog. Cell Metab 14(5):623-634.

30. Urrutia H, Aleman A, \& Eivers E (2016) Drosophila Dullard functions as a Mad phosphatase to terminate BMP signaling. Sci Rep 6:32269.

31. Lu D, et al. (2018) The Ubx Polycomb response element bypasses an unpaired Fab-8 insulator via cis transvection in Drosophila. PLoS One 13(6):e0199353. 


\section{Figure legends}

Figure 1. UCP4b is induced by cold in a cell-autonomous fashion and protects against cold. (A) 1-week old adult flies were exposed to cold $\left(14^{\circ} \mathrm{C}\right)$ for different durations (4 hours, 1 day, and 4 days) followed by whole-body RNA extraction and qPCR $(n=3)$. Transcript levels, normalized to $a$-tubulin, are expressed relative to their levels at $25^{\circ} \mathrm{C}$. Error bars $=\mathrm{SD}$; two-tailed unpaired t-test is performed to measure statistical significance $\left[{ }^{*}(P<0.05) ;{ }^{* *}(P<0.01)\right.$; $\left.{ }^{* * *}(\mathrm{P}<0.001)\right]$. (B) UCP4b was overexpressed with the ubiquitous Act-GeneSwitch (ActGS) driver that is inducible by the drug RU486 (ethanol is the vehicle control), and the cold tolerance of flies treated with RU486 and ethanol control was compared. (C) UCP4b was knocked down in adult flies under the control of ActGS. Flies were exposed to $4^{\circ} \mathrm{C}$ for 6 days and then were brought back to $25^{\circ} \mathrm{C}$. The survivors were scored after 4 hours. Error bars= SD; two-tailed unpaired t-test is performed to measure statistical significance. (D) $3^{\text {rd }}$ instar Drosophila larvae were exposed to cold $\left(14^{\circ} \mathrm{C}\right)$ for 6 hours followed by whole-body RNA extraction and qPCR $(n=3)$. (E) $3^{\text {rd }}$ instar Drosophila larvae were exposed to cold $\left(14^{\circ} \mathrm{C}\right)$ for 4 hours. Then the fat body, brain, gut, and body wall were dissected followed by RNA extraction and qPCR. (F) Drosophila S2R+ cells were exposed to cold $\left(4^{\circ} \mathrm{C}\right)$ at different time points. Then RNA was extracted and qPCR was performed $(n=3)$. Transcript levels are normalized to a-tubulin. $(G)$ $\mathrm{S} 2 \mathrm{R}+$ cells stably transfected with MT-UCP4b plasmid were treated with copper sulfate or mock-treated for 48 hours. Then they were treated with oligomycin A, stained with TMRM, and images were collected. Representative images are shown here. $(\mathrm{H})$ ActGS $>U C P 4 b$ adult flies put on high-sugar food (15\% w/v added sucrose) containing either RU486 or ethanol control for 10 days. Whole-body triglyceride (TAG) levels were measured in male flies $(n=3,8$ adult males per replicate). The TAG levels are normalized to the number of flies. TAG levels are expressed relative to the values in flies on control food.

\section{Figure 2. Calcium signaling and Spargel (SrI) are required for cold-mediated UCP4b} induction. (A) The fat body was dissected in Schneider media at room temperature from $3^{\text {rd }}$ instar Drosophila larvae expressing calcium-sensitive GCaMP reporter under fat body-specific FB-Gal4 driver. Then cold $\left(4^{\circ} \mathrm{C}\right)$ Schneider media was added and the changes in calcium levels were recorded under a microscope as a movie. The rise in GCaMP activity was initiated in multiple parts of the fat body and propagated in the form of waves to other parts. The images represent the temporal progression of the waves (see Supplementary Movie S1). The yellow arrows indicate locations with differential GCaMP activity in different timeframes (B) S2R+ cells were pre-incubated with calcium chelator BAPTA-AM before they were exposed to cold treatment $\left(4^{\circ} \mathrm{C}\right.$ for 1 hour). Then RNA was extracted and qPCR was performed; $(n=3$, normalized to a-tubulin). The relative $U C P 4 b$ expression is compared to control pre-incubated with DMSO. (C) Drosophila S2R+ cells were pre-treated with $10 \mathrm{ug} / \mathrm{mL}$ cycloheximide (CHX) or mock-treated for 4 hours and then exposed to cold $\left(4^{\circ} \mathrm{C}\right)$ for 2 hours. Then RNA was extracted and qPCR was performed. (D) Drosophila S2R + cells were pre-treated with $10 \mathrm{ug} / \mathrm{mL}$ actinomycin $D(A c t D)$ or mock-treated for 4 hours and then exposed to cold $\left(4^{\circ} \mathrm{C}\right)$ for 2 hours. Then RNA was extracted and qPCR was performed $(n=3)$. Transcript levels are normalized to a-tubulin. (E, F) Srl was knocked down in S2R+ cells by dsRNA treatment, then the cells were exposed to cold $\left(4^{\circ} \mathrm{C}\right.$ for 1 hour). RNA was extracted and UCPb and $\mathrm{Srl}$ transcript levels were measured by qPCR ( $n=3$, normalized to a-tubulin). (G) tubGal4, tubGal80 ${ }^{t s}$; UAS-Srl larvae were exposed to a permissible temperature $\left(29^{\circ} \mathrm{C}\right)$ for 2 days to allow UAS transgene expression and then UCP4b expression was compared with similarly treated tubGal4, tubGal80 ${ }^{t s}$ larvae. $(\mathrm{H}) \mathrm{Srl}$ was knocked down in adult flies with ActGS driver, and survivors were counted after exposure to $4^{\circ} \mathrm{C}$ following the protocol described in Fig $1 \mathrm{C}$. 
Figure 3. BMP signaling suppresses UCP4b expression. (A) Gbb, Sax, Tkv, Put, MAD, Med were knocked down in $\mathrm{S} 2 \mathrm{R}+$ cells with dsRNAs against these genes. The cells were incubated with these dsRNAs for 5 days at $25^{\circ} \mathrm{C}$ and then RNA was extracted and qPCR was done. Transcript levels are normalized to a-tubulin, and UCP4b transcript levels in these conditions are expressed relative to their levels with control dsRNA (targeting GFP) treatment. (B) UCP4b transcript levels in S2R+ cells are treated with $10 \mathrm{uM} \mathrm{LDN}-212854$ (at $25^{\circ} \mathrm{C}$ ) for different durations relative to DMSO treated cells. Transcript levels are normalized to a-tubulin. (C) $U C P 4 b$ transcript levels in S2R+ cells that were pre-treated with cycloheximide (CHX) or mocktreated for 4 hours followed by LDN-212854 treatment (at $25^{\circ} \mathrm{C}$ ) for 4 hours. (D) UCP $4 b$ transcript levels in young adult males on food containing LDN-212854 compared to food containing solvent (DMSO). (E) tubGal4, tubGal80 ${ }^{t s}$; UAS-MAD larvae were exposed to a permissible temperature $\left(29^{\circ} \mathrm{C}\right)$ for 2 days to allow UAS transgene expression. Then they were exposed to cold $\left(14^{\circ} \mathrm{C}\right.$ for 4 hours). Finally, UCP $4 b$ expression was compared with similarly

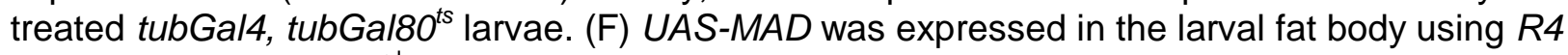
driver. Fat body from $3^{\text {rd }}$ instar larvae at $25^{\circ} \mathrm{C}$ was dissected and transcript levels were measured. (G) S2R+ cells were treated with $M A D$ and/or $S r l$ dsRNA for 5 days at $25^{\circ} \mathrm{C}$ and then $U C P 4 b$ transcript level was measured by qPCR. $(\mathrm{H}) \mathrm{S} 2 \mathrm{R}+$ cells were treated with $M A D$ and Med dsRNAs for 5 days followed by cold treatment $\left(4^{\circ} \mathrm{C}\right.$ for 1 hour) and Srl transcripts were measured. Transcript levels are normalized to a-tubulin.

Figure 4. Cross-talk between cold and BMP signaling. (A) $S 2 R+$ cells are transfected with different plasmid combinations (1: Act-Gal4 + UAS-MAD-FLAG; 2: Act-Gal4 + UAS-MAD-FLAG + Act-Tkv; 3: Act-Gal4 + UAS-MAD-FLAG + Act-Tkv ${ }^{\text {act }}$ ) followed by treatment with either cold or LDN-212854. phospho-MAD, MAD-FLAG, and $\alpha$-tubulin protein levels were determined using western blots. (B) Drosophila S2R+ cells were pre-treated with LDN-212854 (LDN) or mocktreated for 4 hours and then exposed to cold $\left(4^{\circ} \mathrm{C}\right)$ for 2 hours. Then RNA was extracted and qPCR was performed $(n=3)$. UCP $4 b$ transcript levels are normalized to a-tubulin. (C) S2R+ cells were treated with $M A D$ dsRNA for 5 days followed by cold treatment $\left(4^{\circ} \mathrm{C}\right)$ for different durations and UCP $4 b$ transcripts were measured. Transcript levels are normalized to a-tubulin. $(D, E)$ Percentage enrichment for MAD binding upstream of UCP $4 b$ inferred by ChIP with anti-FLAG antibody. (D) MAD-binding in S2R+ cells in response to cold exposure and LDN-212854 treatment is expressed relative to MAD-binding in vehicle-treated (DMSO) cells at $25^{\circ} \mathrm{C}$. (E) MAD-binding in response to cold in the presence/absence of Srl knockdown. (F) Cold sensitivity in adult flies after putting them on food containing LDN-212854 and/or Srl knockdown measured following the protocol described in Fig. $1 \mathrm{C}$. (G) Model of UCP4b regulation by cold and BMP signaling: At normal temperature, MAD, acting downstream of BMP signaling, bind to the upstream region of $U C P 4 b$ and suppress its transcription. Cold exposure leads to the displacement of MAD in an Srl-dependent manner resulting in UCP4b induction. 

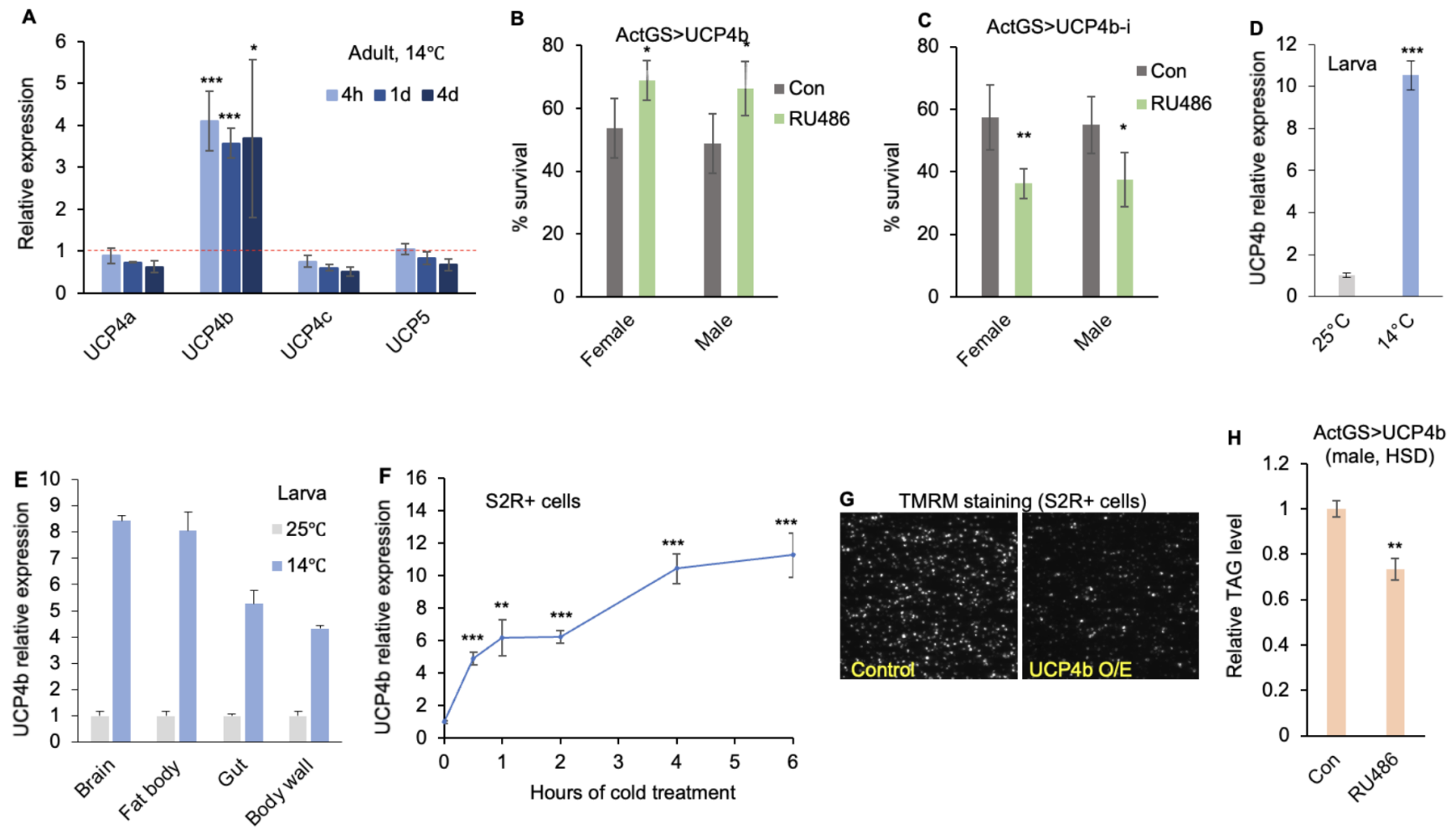

Figure 1 

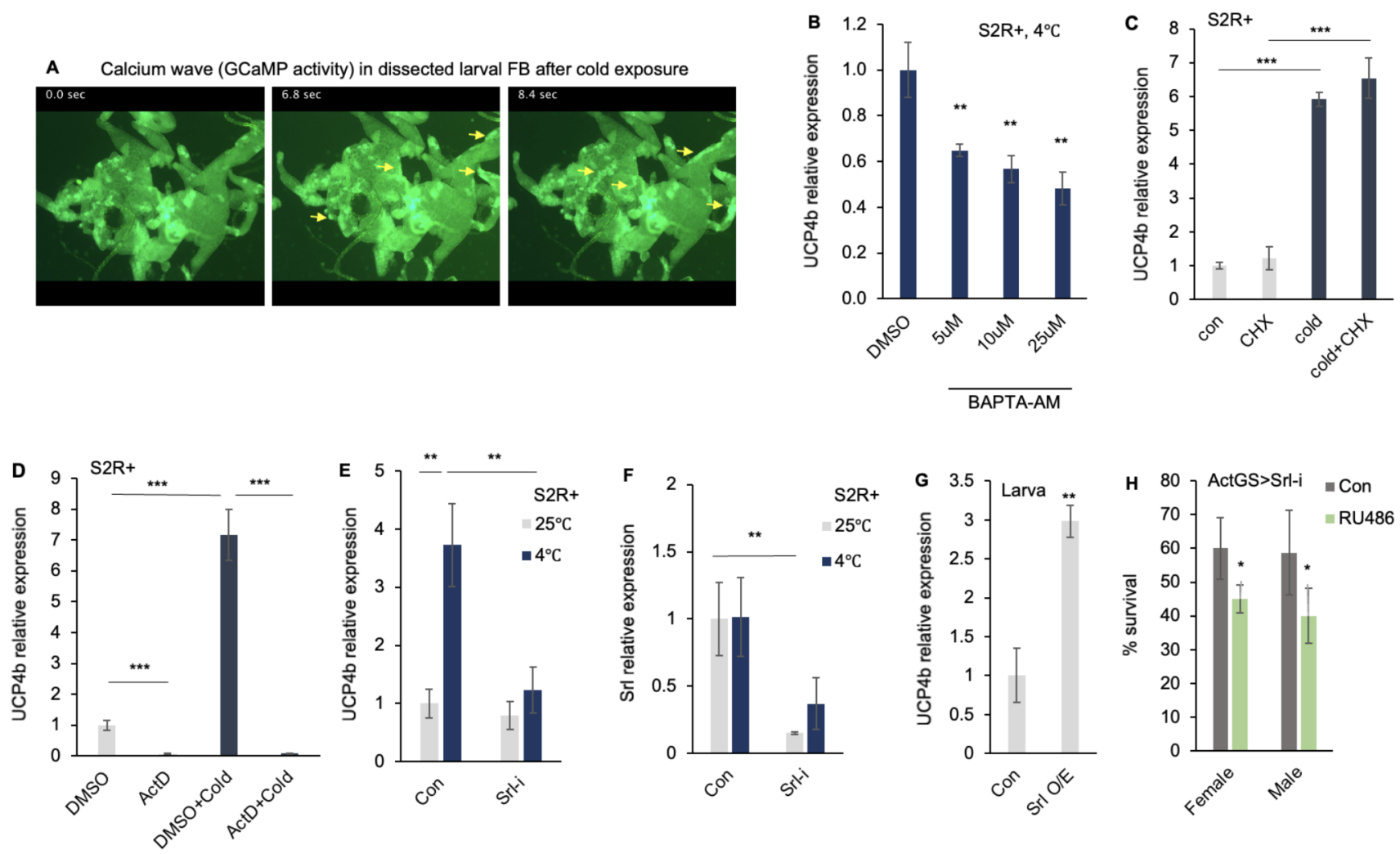

Figure 2 

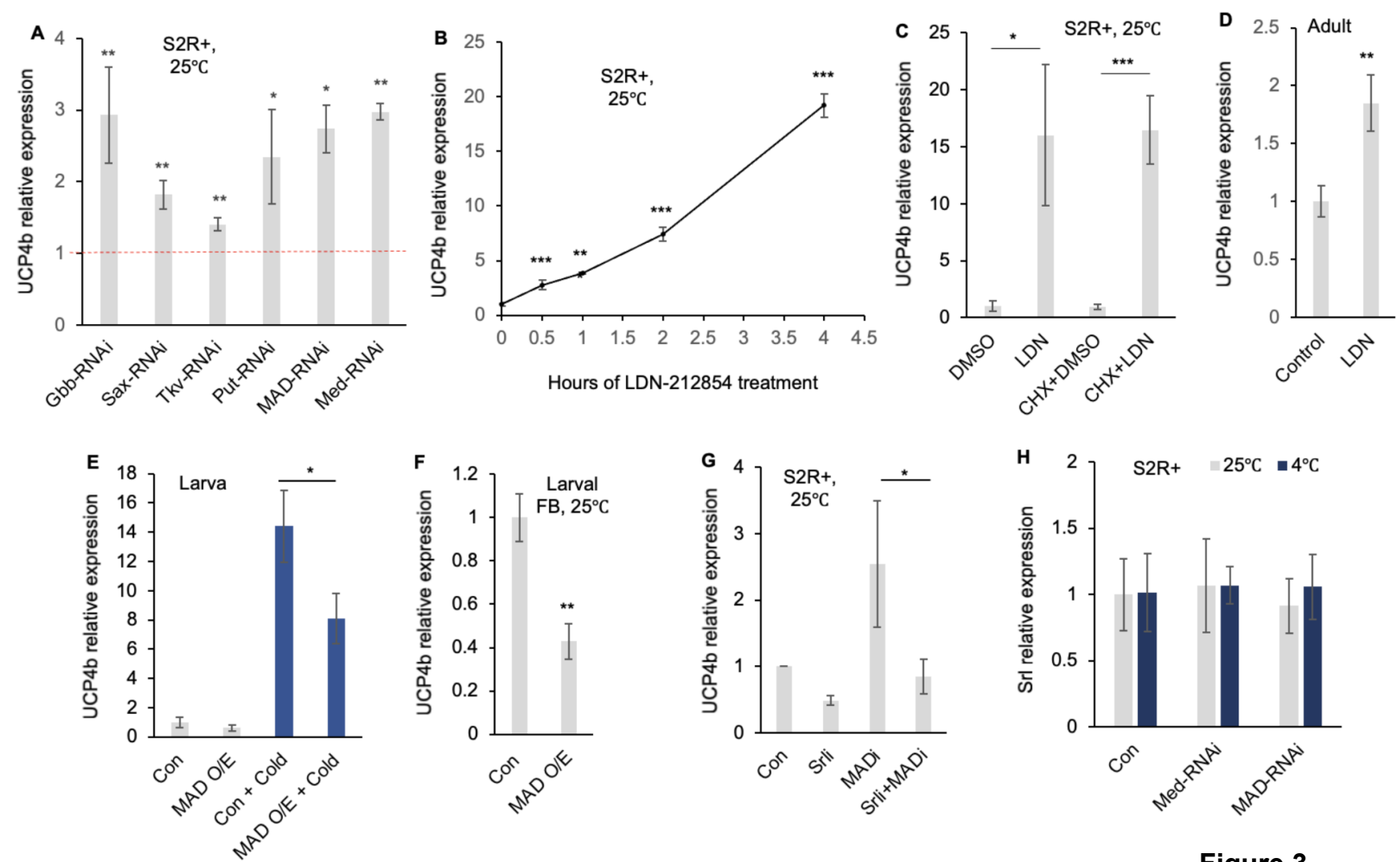

Figure 3 
A

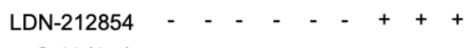

Cold $\left(4^{\circ} \mathrm{C}\right) \quad-\quad-+++-\quad-$

Control

Act-Tkvact + Act-Gal4 + UAS-MAD-FLAG

Act-Tkv + Act-Gal4 + UAS-MAD-FLAG

Act-Gal4 + UAS-MAD-FLAG
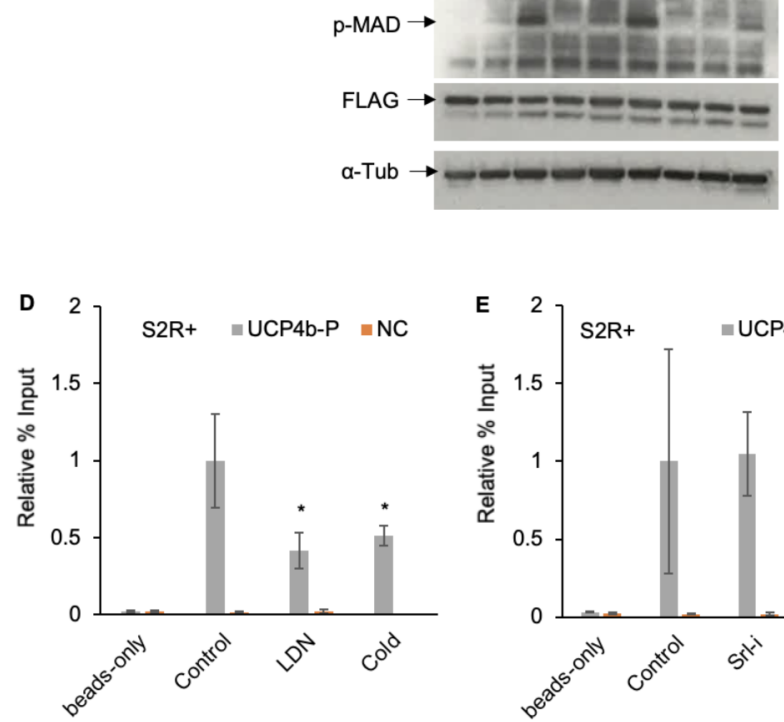
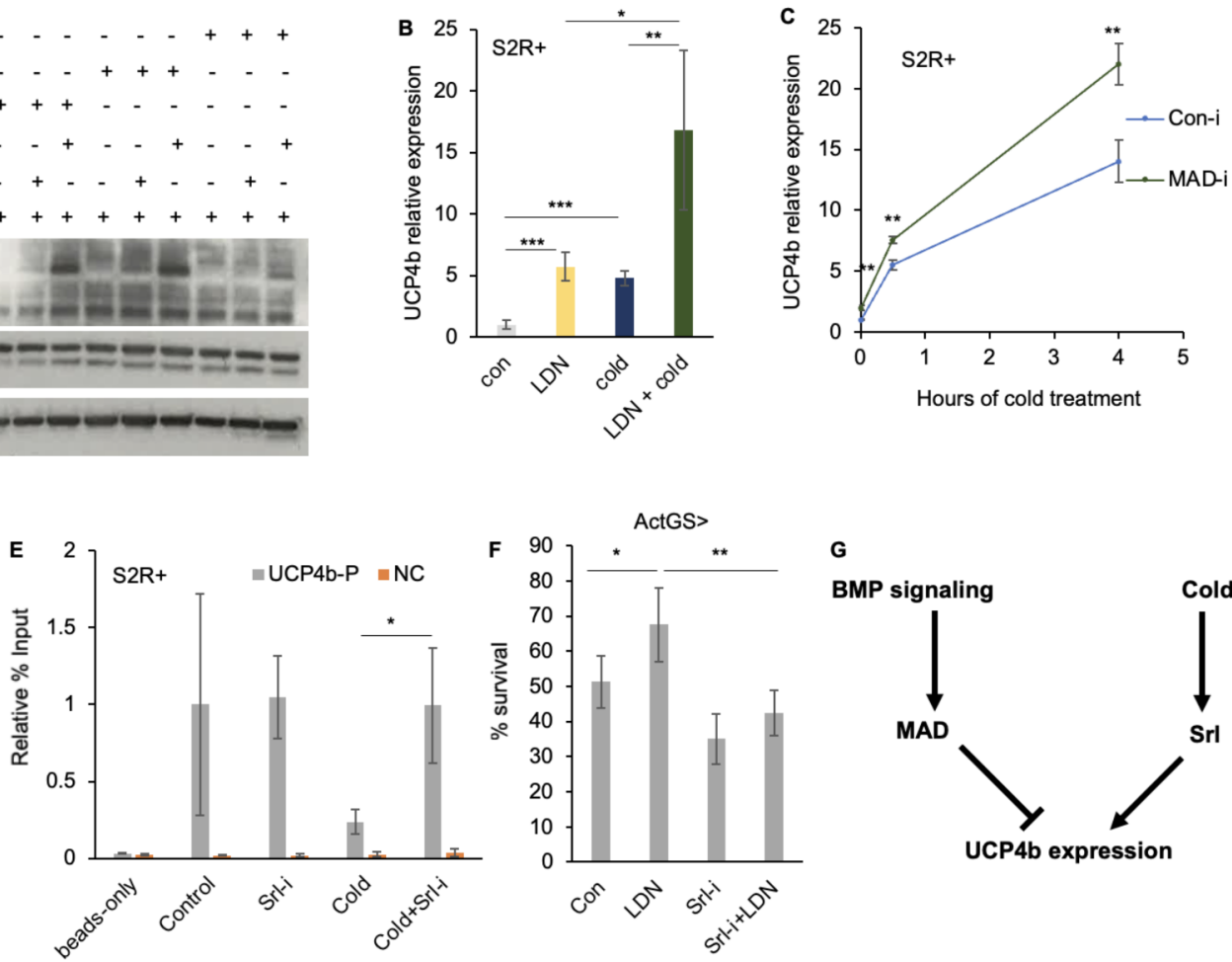

\section{BMP signaling}
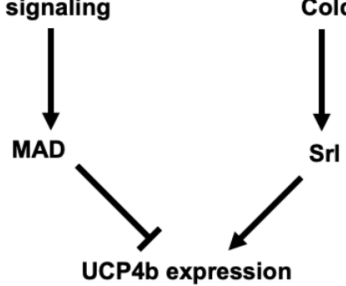

Figure 4 